\title{
Évaluation de la fiabilité d'un dispositif d'évaluation des habiletés sociales chez des adolescents ayant des troubles du comportement ${ }^{1}$
}

Eric Frenette

Université Laval

\author{
Marie-Hélène Hébert et Égide Royer \\ Université Laval
}

\author{
Martin Gendron \\ Université du Québec à Rimouski (campus Lévis) \\ Céline Morand \\ Centre de santé de Portneuf
}

\begin{abstract}
Les troubles du comportement (TC) chez les jeunes d'âge scolaire constituent une préoccupation de plus en plus importante pour les acteurs des systèmes éducatifs québécois et étrangers. Les facteurs de risque pouvant contribuer au développement des TC seraient nombreux (Kauffman, 2005). Parmi ceux-ci figureraient un ensemble d'habiletés liées à la compétence sociale (Bowen, Desbiens, Martin, \& Hamel, 2001). La présente étude vise à éprouver, au moyen de la théorie de la généralisabilité, la fiabilité d'un dispositif d'évaluation conçu pour apprécier le niveau d'habiletés sociales d'adolescents TC selon différents indicateurs du niveau d'habiletés sociales (coopération, contrôle de soi, affirmation de soi), différentes sources d'information (adolescent, parent, intervenant scolaire) et différents temps de mesure (prétest, post-test1, post-test2). Le coefficient de généralisabilité relative se révélant supérieur à la limite jugée satisfaisante, le dispositif d'évaluation se veut assez fiable pour assurer la différenciation d'adolescents TC en regard de leur niveau d'habiletés sociales. Afin de dégager des pistes d'intervention auprès d'adolescents TC, un examen des sources de variation susceptibles d'influencer la précision de la mesure fournie par le dispositif d'évaluation est mené au moyen d'une analyse de facettes (Bertrand, \& Blais, 2004) et d'une étude des composantes de variance les plus significatives (Bain, \& Pini, 1996).
\end{abstract}

Mots-clés : généralisabilité, habiletés sociales, adolescent

Les troubles du comportement chez les jeunes d'âge scolaire constituent une préoccupation de plus en plus importante parmi les acteurs des systèmes d'éducation québécois et étrangers. Les recherches indiquent d'ailleurs qu'un plus grand nombre d'élèves manifestent des troubles du comportement à l'école qu'auparavant (American Psychiatric Association, 1994; Gagnon, Boisjoli, Gendreau \& Vitaro, 2006; Gendron, Royer, Bertrand \& Potvin, 2005; Kauffman, 2005). En effet, selon Kauffman (2005), la prévalence des difficultés de comportement aux États-Unis s'établirait entre 3 et $6 \%$ dans la population scolaire. Au Québec, depuis que le ministère de l'Éducation (MEQ) a regroupé, en 2000, les élèves en difficulté d'apprentissage et de comportement sous la classification « élèves à risque », il est difficile d'estimer le

Eric Frenette, Département des fondements et pratiques en éducation, Université Laval; Martin Gendron, Département des sciences de l'éducation, Université du Québec à Rimouski (campus Lévis); Marie-Hélène Hébert, Département des fondements et pratiques en éducation, Université Laval; Égide Royer, Département d'études sur l'enseignement et l'apprentissage, Université Laval; et Céline Morand, Centre de santé de Portneuf.

Note des auteurs : Les auteurs aimeraient remercier les évaluateurs dont les commentaires et les suggestions ont permis d'améliorer cet article.

Toute correspondance concernant le présent article doit être adressée à Eric Frenette, Département des fondements et pratiques en éducation, Université Laval, bureau 454 TSE, Université Laval, 2320, rue des Bibliothèques, Québec (Québec) G1V 0A6, Canada. Courriel : Eric .Frenette@fse.ulaval.ca nombre réel d'élèves présentant des troubles du comportement (Goupil, 2007). Selon le MEQ (1999), 2,4 \% de la population des élèves d'âge scolaire présenteraient des troubles du comportement, tandis que Vitaro et coll. (1994 dans Saint-Laurent, 2008) affirment que de 6 à $12 \%$ des garçons et que de 3 à $5 \%$ des filles présentent des difficultés comportementales de type extériorisé. Malgré des proportions qui tendent à augmenter d'année en année, ces jeunes sont sous-identifiés par les milieux scolaires (Fortin, Royer, Marcotte \& Potvin, 2000; Kauffman, 2005; Tremblay, 1998).

Plusieurs chercheurs et praticiens utilisent la théorie de l'apprentissage social de Bandura (1986) pour tenter d'expliquer le processus et les déterminants pouvant influencer le développement de troubles du comportement chez les jeunes. Les facteurs de risque pouvant concourir au développement de ces troubles chez les jeunes d'âge scolaire seraient multiples (Gendron, Royer, Bertrand, Potvin \& Frenette, 2006; Kauffman, 2005; Walker, Colvin \& Ramsey, 1995). Ces nombreux facteurs peuvent être regroupés en trois grandes catégories : les caractéristiques de l'environnement (famille, école, communauté, société), les caractéristiques du comportement et les caractéristiques personnelles de l'individu (Cloutier, 1996; Gendron, Royer, Potvin \& Bertrand, 2003; Vitaro \& Gagnon, 2000). Inspirés de la théorie de Bandura (1986), ces regroupements de facteurs de risque

\footnotetext{
${ }^{1}$ Cette recherche a été rendue possible grâce à une subvention du Fonds de recherche sur la société et la culture (FQRSC), dans le cadre du Programme d'établissement de nouveaux professeurs-chercheurs.
} 
illustrent bien l'importance de l'interaction entre les variables associées à l'individu et celles de son environnement dans le développement intégral de la personne. De plus, le choix délibéré d'aborder la présente étude sous l'angle de la théorie de l'apprentissage social de Bandura (1986) répondait à la prémisse que l'efficacité accrue de l'entraînement aux habiletés sociales passe par une approche multimodale où « l'ensemble du milieu de vie du jeune (et en particulier sa famille) s'engage dans le processus éducatif » (Bowen, Desbiens, Gendron \& Bélanger, 2006, p. 216). Le choix méthodologique exposé ultérieurement à propos de l'utilisation du multisource dans le processus d'évaluation des habiletés sociales répondait aussi à cette même logique, basée sur les écrits et des expériences empiriques (Goldstein \& McGinnis, 1997; Gresham \& Elliott, 1990).

Parmi les facteurs de risque associés aux troubles du comportement figurant le plus souvent dans la littérature contemporaine, on retrouve un ensemble d'habiletés sociales qui représentent une partie de la compétence sociale (Bowen et al., 2006; Bowen, Desbiens, Martin \& Hamel, 2001). Selon ces auteurs, il existe bon nombre de programmes d'intervention en milieu scolaire ayant pour but la prévention ou l'intervention auprès d'élèves ayant des troubles du comportement. Ces programmes d'intervention visent précisément à pallier des lacunes importantes sur le plan des habiletés sociales. Des exemples de programmes incluent le PACTE, de Doucette et Fowler (1997), le PARC, de Potvin et al. (1995), le PEC, de Gendron, Royer et Morand (2005), Prends le volant, de Potvin et al. (1994), et Skillstreaming the Adolescent, de Goldstein et McGinnis (1997).

Malgré la popularité et le potentiel certain des programmes d'entraînement aux habiletés sociales, leur efficacité demeure modeste (Gresham, 1998; Walker et al., 1995). Aux raisons évoquées pour tenter d'expliquer cette situation (Gresham, 1998), on peut ajouter l'identification imprécise des besoins des élèves en matière d'habiletés sociales, la rareté des outils de mesure spécifiques pour y arriver, ainsi que l'absence d'une évaluation rigoureuse dans le temps (Cartledge \& Milburn, 1995; Flanagan, Alphonso, Primavera, Povall \& Higgins, 1996). Le Questionnaire d'évaluation des habiletés sociales (Fortin, Royer, Marcotte, Potvin \& Joly, 2001; traduction du Social Skills Rating System SSRS -, de Gresham \& Elliott, 1990) est l'un des rares outils permettant de mesurer le niveau d'habiletés sociales, et cela, avec la possibilité d'avoir recours à trois sources différentes d'information : l'élève, le parent, l'intervenant scolaire ${ }^{2}$ ) (Gendron et al., 2005; Junttila, Voeten, Kaukiainen \& Vaurus, 2006; Warnes, Sheridan, Geske \& Warnes, 2005). Le SSRS constitue une référence dans le domaine de l'évaluation de la compétence sociale et des habiletés sociales (DiPerna \& Volpe, 2005; Flanagan et al., 1996; Kauffman, 2005; Warnes et al., 2005). Nouvellement validées auprès d'un échantillon québécois, les diverses versions du questionnaire (c.-à-d., Adolescent autorévélé, Parent, Intervenant scolaire), lorsque utilisées conjointement, fournissent tant aux chercheurs qu'aux praticiens de l'information précieuse leur permettant de mieux cibler les moyens et les stratégies de l'intervention (programmes d'entraînement aux habiletés sociales) et d'en mesurer l'efficacité. Il paraissait donc important d'effectuer une étude visant à évaluer la pertinence de recourir à un dispositif d'évaluation des habiletés sociales (coopération, contrôle de soi, affirmation de soi) d'adolescents à plusieurs temps de mesure (prétest, postest 1 , postest 2 ) et au moyen de différentes sources d'information (adolescent, parent, intervenant scolaire). En quoi l'utilisation d'un tel dispositif, plutôt que de recourir à un seul temps de mesure et à une seule source d'information, permettrait d'obtenir une plus-value dans l'évaluation du profil d'adolescents en matière d'habiletés sociales en vue d'assurer un meilleur suivi de l'intervention? Pour répondre à cette question, nous avons eu recours à la théorie de la généralisabilité.

\section{Théorie de la généralisabilitée ${ }^{3}$}

Dans un cadre où les ressources seraient illimitées, il serait concevable d'avoir recours à tous les indicateurs du niveau d'habiletés sociales imaginables, à toutes les sources d'information admissibles, et ce, à plusieurs reprises afin de déterminer avec exactitude le niveau réel d'habiletés sociales d'adolescents ayant des troubles du comportement. Pour diverses raisons, une telle approche ne peut être mise en pratique. Il faut plutôt se contenter de déterminer, au moyen d'une étude de généralisabilité (Bain \& Pini, 1996; Cardinet \& Tourneur, 1985), si le niveau moyen d'habiletés sociales observé par l'entremise d'un nombre limité d'indicateurs du niveau d'habiletés sociales, de sources d'information et de temps de mesure, est généralisable à tous les indicateurs du niveau d'habiletés sociales admissibles, à toutes les sources d'information concevables et à un nombre infini de temps de mesure. Dans l'affirmative, il sera alors possible de certifier que le niveau moyen d'habiletés sociales observé, moyennant quelques indicateurs du niveau d'habiletés sociales, quelques sources d'information sélectionnées et un nombre restreint de temps de mesure, traduit le niveau réel d'habiletés sociales d'adolescents ayant des troubles du comportement.

Pour savoir si effectivement les résultats observés au moyen de quelques indicateurs du niveau d'habiletés sociales, de quelques sources d'information et de quelques temps de mesure sont généralisables à tous les indicateurs du niveau d'habiletés sociales admissibles, à toutes les sources d'information imaginables et à tous les temps de mesure souhaitables, un coefficient de généralisabilité doit être calculé. Pour chaque étude, selon l'intention de la mesure, un choix d'indice doit néanmoins s'imposer : le coefficient de généralisabilité dite « relative » s'emploie pour hiérarchiser ou comparer entre eux les différents objets de mesure (par ex., : Quel adolescent présente le niveau le plus élevé d'habiletés sociales?), tandis que le coefficient de généralisabilité dite « absolue » s'utilise pour comparer les scores observés selon un standard quelconque (par ex., : Quels adolescents présentent un score supérieur à 45 au SSRS?) (Bain \& Pini, 1996). Dans le cadre de la présente étude, le coefficient de généralisabilité relative a été utilisé, car il s'agissait de vérifier si la hiérarchisation des adolescents en fonction de leurs habiletés sociales était similaire selon les différents indicateurs du niveau d'habiletés sociales, temps de mesure et sources d'information.

\section{Objectif de la recherche}

La présente étude visait à éprouver, au moyen de la théorie de la généralisabilité, la fiabilité d'un dispositif d'évaluation conçu

\footnotetext{
${ }^{2}$ Dans le cadre de cette étude, le terme « intervenant scolaire » comprend des enseignants titulaires de la classe, des enseignants non titulaires de la classe, des professionnels (par ex., psychologues, psychoéducateurs, travailleurs sociaux) et des techniciens en éducation spécialisée.

${ }^{3}$ Pour de plus amples renseignements sur la théorie de la généralisabilité, se référer à Bertrand (1988) et Bertrand et Blais (2004).
} 
pour déterminer le niveau d'habiletés sociales d'adolescents ayant des troubles du comportement (A) selon différents indicateurs du niveau d'habiletés sociales (I; coopération, contrôle de soi, affirmation de soi), différentes sources d'information ( $\mathrm{S}$; adolescent lui-même, parent et intervenant scolaire) et différents temps de mesure ( $\mathrm{T}$; prétest, postest 1, postest 2).

\section{Méthodologie}

\section{Le questionnaire}

Les habiletés sociales d'adolescents ayant des troubles du comportement sont évaluées au moyen des versions « Adolescent autorévélé » (39 items), « Parent » (52 items) et « Enseignant » 4 (42 items) du Questionnaire d'évaluation des habiletés sociales (Fortin et al., 2001; traduction du Social Skills Rating System SSRS -, de Gresham \& Elliott, 1990). Ce questionnaire permet d'évaluer cinq sous-échelles d'habiletés sociales, soit a) la coopération (par ex., : Je respecte les consignes de l'enseignante); b) l'affirmation de soi (par ex., : J'initie des conversations avec mes compagnons de classe); c) l'empathie (par ex., : J'écoute mes amis lorsqu'ils ont des problèmes); d) le contrôle de soi (par ex., : Je termine calmement les chicanes avec mes parents), et e) la responsabilisation (par ex., : L'élève suit les règles lorsqu'il joue avec les autres). La fidélité et la validité de chacune des sous-échelles sont documentées dans le manuel du SSRS (Gresham \& Elliot, 1990). La somme de ces sous-échelles constitue le score total représentant le niveau d'habiletés sociales des adolescents. Puisque les souséchelles « empathie » et « responsabilisation» ne s'adressent qu'aux adolescents et qu'aux parents respectivement, et qu'elles s'en trouvent par conséquent non généralisables à toutes les sources d'information dans le dispositif d'évaluation utilisé, elles ont été retirées des analyses de généralisabilité de la présente étude. La moyenne, l'écart-type et le niveau de consistance interne pour chacun des indicateurs du niveau d'habiletés sociales, à chacun des temps de mesure et pour chacune des sources d'information sont présentés au Tableau 1. Les niveaux de consistance interne ont été jugés acceptables à satisfaisants, variant de 0,62 à $0,90(M \alpha=0,78, E$. $-T . \alpha=0,07)$.

\section{La collecte des données}

La collecte des données a eu lieu en novembre 2000, en mars 2001 et en juin 2001 dans trois écoles de la grande région de Québec. Une intervention auprès d'adolescents ayant des troubles du comportement a été mise en œuvre entre le premier (prétest) et le second (postest 1) temps de mesure. Cette intervention, ayant pour but d'améliorer les habiletés sociales, a pris la forme d'une série de 20 ateliers d'entraînement aux habiletés sociales en classe sur une période de 20 semaines (Gendron, Royer \& Morand, 2005). La compétence sociale d'adolescents ayant été formellement jugés par le ministère de l'Éducation, du Loisir et du Sport du Québec comme ayant des troubles du comportement (code 12, maintenant classifié dans la catégorie «élèves à risque »; voir la définition et les critères de détermination dans MEQ, 2000) a été appréciée par trois sources d'information : l'adolescent lui-même $(n=49)$, un parent $(n=39)$ et un intervenant scolaire $(n=45)$. La participation des adolescents, des parents et des intervenants scolaires s'est faite sur une base volontaire. Les adolescents ont rempli le Questionnaire d'évaluation des habiletés sociales durant les heures de classe, les parents, à la maison, et les enseignants, lors de périodes libres durant les heures de travail.

\section{L'échantillon retenu}

Le logiciel EduG (version 1.3) spécialisé en théorie de la généralisabilité ne permettant pas l'emploi de devis non balancés, l'étude reposait sur les 23 adolescents ayant des troubles du comportement (16 garçons et 7 filles), âgés de 12 à 15 ans ( 3 de 12 ans, 8 de 13 ans, 11 de 14 ans et 1 de 15 ans). Pour chacun des adolescents, des indicateurs du niveau d'habiletés sociales (coopération, contrôle de soi, affirmation de soi) ont été appréciés par chacune des sources d'information (l'adolescent lui-même, un parent et un intervenant scolaire), et ce, à chacun des temps de mesure (prétest, postest 1, postest 2).

\section{La démarche d'analyse}

Afin d'évaluer les qualités métrologiques du dispositif d'évaluation à quatre facettes croisées constitué, les données ont été analysées selon la théorie de la généralisabilité au moyen du logiciel EduG (version 1.3). Les observations collectées ont été structurées suivant les plans d'observation, d'estimation et de mesure suivants.

Plan d'observation. Dans la situation de mesure à l'étude, on a distingué quatre facettes : Adolescents ayant des troubles du comportement (A), Indicateurs du niveau d'habiletés sociales (I), Sources d'information (S) et Temps de mesure (T). On a dénombré 23 niveaux observés pour la facette Adolescents ayant des troubles du comportement (23 adolescents ayant des troubles du comportement pour lesquels chacun des indicateurs du niveau d'habiletés sociales a été apprécié par chacune des sources d'information, et ce, à chacun des temps de mesure), 3 niveaux observés pour la facette Indicateurs du niveau d'habiletés sociales (coopération, contrôle de soi, affirmation de soi), 3 niveaux observés pour la facette Sources d'information (adolescent, parent, intervenant scolaire) et 3 niveaux observés pour la facette Temps de mesure (prétest, postest 1, postest 2). Comme le plan d'observation d'une étude de généralisabilité demande entre autres à ce que les facettes soient bien établies et les niveaux observés, bien dénombrés, ces informations ont été synthétisées dans les trois premières colonnes du Tableau 2.

$\mathrm{Au}$ chapitre des relations entre les facettes, puisque le niveau d'habiletés sociales de chacun des 23 adolescents a été apprécié par chacune des trois sources d'information, au moyen des trois indicateurs du niveau d'habiletés sociales à chacun des trois temps de mesure, la facette Adolescents ayant des troubles du comportement (A) a été « croisée » avec les facettes Sources d'information $(A \times S)$, Indicateurs du niveau d'habiletés sociales $(\mathrm{A} \times \mathrm{I})$ et Temps de mesure $(\mathrm{A} \times \mathrm{T})$. Étant donné que chacun des trois indicateurs du niveau d'habiletés sociales a été apprécié par chacune des trois sources d'information à chacun des trois temps de mesure, la facette Indicateurs du niveau d'habiletés sociales (I) a été, quant à elle, « croisée » avec les facettes Sources d'information $(I \times S)$ et Temps de mesure $(\mathrm{I} \times \mathrm{T})$. Enfin, puisque chacune des trois sources d'information a été

\footnotetext{
${ }^{4}$ La version « enseignant» du Questionnaire d'évaluation des habiletés sociales peut être utilisée par un intervenant scolaire (par ex., enseignant, psychologue, psychoéducateur, travailleur social, technicien en éducation spécialisée).
} 
Tableau 1

Statistiques descriptives

\begin{tabular}{|c|c|c|c|c|c|c|c|c|c|}
\hline \multirow[b]{2}{*}{ Facettes } & \multicolumn{3}{|c|}{ Coopération $^{\mathrm{a}}$} & \multicolumn{3}{|c|}{ Contrôle de soi $^{\mathrm{a}}$} & \multicolumn{3}{|c|}{ Affirmation de soi $^{\mathrm{a}}$} \\
\hline & Prétest & Postest 1 & Postest 2 & Prétest & Postest 1 & Postest 2 & Prétest & Postest 1 & Postest 2 \\
\hline \multicolumn{10}{|l|}{ Adolescent } \\
\hline $\mathrm{M}$ & 16,26 & 17,35 & 17,39 & 11,00 & 12,35 & 13,52 & 11,91 & 12,09 & 12,35 \\
\hline É.T. & $(3,82)$ & $(3,58)$ & $(3,88)$ & $(3,52)$ & $(3,41)$ & $(3,13)$ & $(3,03)$ & $(3,15)$ & $(3,30)$ \\
\hline$\alpha$ & 0,80 & 0,70 & 0,73 & 0,75 & 0,81 & 0,79 & 0,62 & 0,72 & 0,64 \\
\hline \multicolumn{10}{|l|}{ Parent } \\
\hline $\mathrm{M}$ & 10,13 & 11,52 & 11,30 & 10,22 & 11,83 & 11,91 & 13,35 & 13,61 & 14,17 \\
\hline É.T. & $(3,38)$ & $(3,60)$ & $(3,91)$ & $(3,29)$ & $(3,68)$ & $(3,36)$ & $(3,81)$ & $(4,27)$ & $(3,88)$ \\
\hline$\alpha$ & 0,69 & 0,80 & 0,81 & 0,80 & 0,81 & 0,81 & 0,76 & 0,81 & 0,79 \\
\hline \multicolumn{10}{|c|}{ Intervenant scolaire } \\
\hline M & 13,22 & 13,83 & 12,35 & 10,13 & 12,74 & 11,65 & 9,61 & 11,52 & 9,78 \\
\hline É.T. & $(3,38)$ & $(3,80)$ & $(4,70)$ & $(4,20)$ & $(4,06)$ & $(4,11)$ & $(4,02)$ & $(4,57)$ & $(4,08)$ \\
\hline$\alpha$ & 0,83 & 0,86 & 0,85 & 0,77 & 0,90 & 0,72 & 0,86 & 0,90 & 0,83 \\
\hline
\end{tabular}

${ }^{a}$ Chacune des sous-échelles d'habiletés sociales (par croisement Sources d'information $\times$ Temps de mesure) est constituée de 10 items cotés sur une échelle à trois points $(0,1,2)$.

sollicitée à chacun des trois temps de mesure, la facette Sources d'information $(S)$ a été « croisée » avec la facette Temps de mesure $(\mathrm{S} \times \mathrm{T})$. Les données exploitées ont été structurées suivant le plan d'observation à quatre facettes croisées $\mathrm{A} \times \mathrm{I} \times \mathrm{S} \times \mathrm{T}$, lequel est représenté visuellement par le diagramme d'Euler-Venn présenté à la Figure 1.

Plan d'estimation. En supposant que les 23 adolescents ayant des troubles du comportement dont les résultats ont été retenus aux fins d'analyse aient été choisis parmi des milliers d'adolescents présentant des troubles du comportement disponibles et que les trois temps de mesure aient été puisés à même des milliers de temps de mesure admissibles, les facettes A et T ont été qualifiées d' « aléatoires infinies ». Les trois indicateurs du niveau d'habiletés sociales étudiés ayant été sélectionnés parmi les cinq indicateurs du Questionnaire d'évaluation des habiletés sociales, il a été choisi de considérer la facette I comme « aléatoire finie ». Enfin, puisqu'il existe d'autres sources d'information aptes à juger du niveau d'habiletés sociales d'adolescents ayant des troubles du comportement, notamment un psychoéducateur ou un psychologue et un travailleur social ou un technicien en éducation spécialisée, il a été décidé de considérer la facette $\mathrm{S}$ comme « aléatoire finie ». Le Tableau 2 rapporte ces informations dans les deux dernières colonnes.

Plan de mesure. Dans notre volonté de différencier les adolescents ayant des troubles du comportement (A) selon leur niveau d'habiletés sociales au moyen des indicateurs du niveau d'habiletés sociales (I), des sources d'information (S) et des temps de mesure (T), le plan de mesure A/IST a été retenu.

\section{Résultats}

Le Tableau 3 synthétise, pour la situation de mesure à l'étude, les résultats de l'étude de généralisabilité obtenus au moyen du logiciel EduG (version 1.3).

Le coefficient de généralisabilité relative $(0,8122)$ étant supérieur à la limite conventionnellement jugée acceptable $(0,80)$, le niveau de précision de la mesure fournie par le dispositif d'évaluation a été considéré comme satisfaisant. Le dispositif d'évaluation était donc assez fiable pour assurer la différenciation des adolescents ayant des troubles du comportement selon leur niveau d'habiletés sociales, sans égard au temps de mesure, à la source d'information ou à l'indicateur du niveau d'habiletés sociales privilégié.

Bien que le coefficient de généralisabilité relative ait été jugé satisfaisant, il a été décidé de procéder à un examen des sources de variation susceptibles d'influencer la précision de la mesure fournie par le dispositif d'évaluation afin de cibler des pistes potentielles d'intervention et de prévention auprès des adolescents ayant des troubles du comportement pour les besoins des milieux d'éducation. Pour ce faire, deux analyses statistiques ont été effectuées : une analyse de facettes (Bertrand \& Blais, 2004) et une étude des composantes de variance les plus significatives (Bain \& Pini, 1996). L'analyse de facettes, le pendant de l'analyse d'items en théorie classique des tests, vise à déterminer les niveaux observés de chacune des facettes qui influencent le plus la précision de la mesure fournie par le dispositif d'évaluation. La procédure utilisée pour l'analyse de facettes consistait à éliminer les niveaux

Tableau 2

Dispositif d'évaluation : plan d'observation et plan d'estimation

\begin{tabular}{|c|c|c|c|c|}
\hline Facettes & Niveaux observés & $\begin{array}{c}\text { Nombre de } \\
\text { niveaux }\end{array}$ & $\begin{array}{c}\text { Niveaux } \\
\text { admissibles }\end{array}$ & Type \\
\hline A & $\mathrm{A}_{1}$ à $\mathrm{A}_{23}$ & 23 & Infini & Aléatoire infinie \\
\hline I & Coopération, contrôle de soi, affirmation de soi & 3 & 5 & Aléatoire finie \\
\hline $\mathrm{S}$ & Adolescent, parent, intervenant scolaire & 3 & 5 & Aléatoire finie \\
\hline $\mathrm{T}$ & Prétest, postest 1 , postest 2 & 3 & Infini & Aléatoire infinie \\
\hline
\end{tabular}




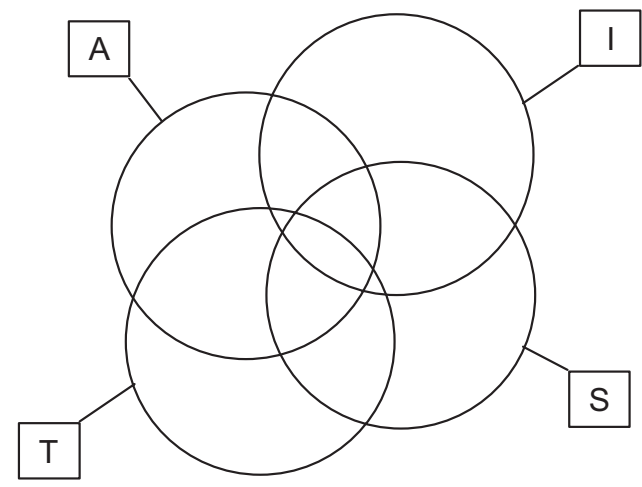

Figure 1. Illustration du plan d'observation à quatre facettes croisées du dispositif d'évaluation $\mathrm{A} \times \mathrm{I} \times \mathrm{S} \times \mathrm{T}$.

observés un à un et à recalculer le coefficient de généralisabilité relative. Les résultats sont présentés au Tableau 4.

En examinant les résultats de l'analyse de facettes, on remarque que le retrait de chacun des trois niveaux observés de la facette Indicateurs du niveau d'habiletés sociales (coopération, affirmation de soi, contrôle de soi) a eu pour effet de diminuer la fiabilité du dispositif d'évaluation. Ce qui justifie la pertinence de recourir aux trois indicateurs du niveau d'habiletés sociales pour apprécier avec précision le niveau d'habiletés sociales d'adolescents ayant des troubles du comportement. Le retrait de chacun des trois niveaux observés de la facette Sources d'information (adolescent, parent, intervenant scolaire) a eu pour effet de diminuer la fiabilité du dispositif d'évaluation. Ce résultat permet de justifier le recours aux trois sources d'information pour évaluer avec précision le niveau d'habiletés sociales d'adolescents ayant des troubles du comportement. Enfin, l'analyse de la facette Temps de mesure a révélé que le retrait du niveau observé « prétest » contribuait à augmenter, bien que légèrement, la précision de la mesure fournie par le dispositif d'évaluation (de 0,8122 à 0,8428). Ce résultat s'explique par la mise en œuvre, entre le premier (prétest) et le second (postest 1) temps de mesure, d'une intervention ayant pour but d'améliorer les habiletés sociales des adolescents de l'échantillon. Par conséquent, le dispositif d'évaluation préconisé dans cette étude s'est révélé sensible aux changements qui peuvent s'opérer dans les habiletés sociales à la suite de la mise en œuvre d'une intervention visant à modifier des comportements chez des adolescents ayant des troubles du comportement.

Tableau 3

Étude de généralisabilité du plan de mesure A/IST

\begin{tabular}{lcccr}
\hline $\begin{array}{c}\text { Sources de } \\
\text { variation }\end{array}$ & Variance vraie & $\begin{array}{c}\text { Sources de } \\
\text { variation }\end{array}$ & $\begin{array}{c}\text { Variance d'erreur } \\
\text { relative }\end{array}$ & $\%$ \\
\hline \multirow{2}{*}{ A } & \multirow{2}{*}{3,5074} & & & \\
& & AT & 0,2088 & 26 \\
& & AS & 0,2971 & 36 \\
& & AI & 0,1526 & 19 \\
& & ATS & 0,0717 & 9 \\
& & ATI & 0,0434 & 5 \\
\multirow{2}{*}{ Totaux } & 3,5074 & ASI & 0,0295 & 4 \\
& & ATSI & 0,0130 & 2 \\
\hline
\end{tabular}

Nota. Coefficient de généralisabilité relative : 0,8122.
Tableau 4

Analyse de facettes

\begin{tabular}{clc}
\hline \multicolumn{1}{c}{ Facettes } & Niveaux observés & Coefficient relatif \\
\hline Indicateurs du niveau & Coopération & 0,7904 \\
d'habiletés sociales & Contrôle de soi & 0,5842 \\
& Affirmation de soi & 0,6807 \\
Sources d'information & Adolescent & 0,7155 \\
& Parent & 0,7064 \\
Temps de mesure & Intervenant scolaire & 0,6079 \\
& Prétest & 0,8428 \\
& Postest 1 & 0,7260 \\
& Postest 2 & 0,7603 \\
\hline
\end{tabular}

Quant aux deux autres niveaux observés de la facette Temps de mesure (postest 1 et postest 2), leur retrait a eu pour effet de diminuer la fiabilité du dispositif d'évaluation.

L'analyse des composantes de variance les plus significatives a permis, quant à elle, de cerner les sources de variation qui affectaient le plus le dispositif d'évaluation et qui ont eu pour effet d'introduire un biais - quoique minime étant donné la valeur considérée comme satisfaisante du coefficient de généralisabilité relative - dans l'évaluation du niveau d'habiletés sociales d'adolescents ayant des troubles du comportement. De par cette analyse, on a constaté que les sources de variation qui concouraient le plus à l'erreur de mesure (voir la dernière colonne du Tableau 3) étaient : 1) AS (36\%; interaction Adolescents ayant des troubles du comportement $\times$ Sources d'information); 2) AT (26\%; interaction Adolescents ayant des troubles du comportement $\times$ Temps de mesure), et 3) AI (19\%; interaction Adolescents ayant des troubles du comportement $\times$ Indicateurs du niveau d'habiletés sociales). Seule l'interaction AS est significative. Pour qu'une source de variation soit considérée comme significative, la valeur de la composante aléatoire (voir la cinquième colonne du Tableau 5) associée à la source de variation doit être plus grande que deux fois l'erreur-type (voir la dernière colonne du Tableau 5) la concernant (Bain \& Pini, 1996).

L'interaction AS (Adolescents ayant des troubles du comportement $\times$ Sources d'information) est représentée graphiquement à la Figure 2. La présence d'une telle interaction signifie que le niveau d'habiletés sociales de certains adolescents ayant des troubles du comportement a été apprécié mieux ou moins bien que prévisible par certaines sources d'information. Par exemple, les intervenants scolaires pourraient avoir eu du mal à apprécier le niveau d'habiletés sociales d'adolescents ayant des troubles légers du comportement, ce qui aurait pu les inciter à évaluer ces adolescents d'une manière différente de leur parent ou des adolescents euxmêmes. L'interaction AT (Adolescents ayant des troubles du comportement $\times$ Temps de mesure) est représentée graphiquement à la Figure 3. La présence d'une telle interaction signifie que le niveau d'habiletés sociales de certains adolescents ayant des troubles du comportement a été apprécié mieux ou moins bien que prévisible à certains temps de mesure. Par exemple, le temps de mesure « postest $2 »$ pourrait avoir fourni une estimation à la hausse du niveau d'habiletés sociales chez les adolescents d'un groupe d'âge particulier, alors qu'il n'aurait pas eu cet effet chez d'autres groupes d'âges. Enfin, l'interaction AI (Adolescents ayant des troubles du comportement $\times$ Indicateurs du niveau d'habiletés sociales) est représentée graphiquement à la Figure 4. La présence 
Tableau 5

Analyse de la variance du dispositif d'évaluation $A \times I \times S \times T$

\begin{tabular}{lrrrrr}
\hline $\begin{array}{c}\text { Sources de } \\
\text { variation }\end{array}$ & $\begin{array}{c}\text { Sommes des } \\
\text { carrés }\end{array}$ & $\begin{array}{c}\text { Degrés de } \\
\text { liberté }\end{array}$ & Carrés moyens & $\begin{array}{c}\text { Composantes } \\
\text { aléatoires }\end{array}$ & Erreurs-types \\
\hline A & 2568,2158 & 22 & 116,7371 & 2,5200 & 1,3050 \\
T & 171,0757 & 2 & 85,5378 & 0,2841 & 0,2991 \\
S & 551,9742 & 2 & 275,9871 & $-0,2787$ & 1,2476 \\
I & 474,4477 & 2 & 237,2238 & $-0,3270$ & 1,1494 \\
AT & 400,2576 & 44 & 9,0968 & $-0,0385$ & 0,2703 \\
AS & 1954,9147 & 44 & 44,4299 & 3,0339 & 1,0585 \\
AI & 708,4412 & 44 & 16,1009 & 0,4800 & 0,4255 \\
TS & 73,5040 & 4 & 18,3760 & 0,1283 & 0,1572 \\
TI & 50,3929 & 4 & 12,5982 & 0,1220 & 0,1088 \\
SI & 1167,5523 & 4 & 291,8881 & 4,0583 & 2,4426 \\
ATS & 805,1626 & 88 & 9,1496 & 1,8790 & 0,4713 \\
ATI & 334,9404 & 88 & 3,8061 & 0,0978 & 0,2262 \\
ASI & 1010,8921 & 88 & 11,4874 & 2,6583 & 0,5842 \\
TSI & 31,1143 & 8 & 3,8893 & 0,0164 & 0,0773 \\
ATSI & 618,2190 & 176 & 3,5126 & 3,5126 & 0,3723 \\
\hline
\end{tabular}

d'une telle interaction signifie que le niveau d'habiletés sociales de certains adolescents a été apprécié mieux ou moins bien que prévisible en fonction de certains indicateurs du niveau d'habiletés sociales. Par exemple, l'indicateur d'habiletés sociales « coopération » pourrait avoir fourni une sous-estimation du niveau d'habiletés sociales chez les adolescentes ayant des troubles du comportement, alors qu'il n'aurait pas eu cet effet chez les adolescents.

\section{Discussion}

Les résultats de l'étude de généralisabilité (coefficient de généralisabilité relative satisfaisant), de l'analyse de facettes (apport significatif des niveaux observés des facettes I, S et T) et de l'étude des composantes de variance les plus significatives (mise en évidence d'un effet d'interaction significatif AS) confirment l'importance de recourir à un dispositif d'évaluation comportant plusieurs indicateurs du niveau d'habiletés sociales, temps de mesure et sources d'information pour obtenir une évaluation précise du niveau d'habiletés sociales d'adolescents ayant des troubles du comportement.

Face à ce constat, on peut proposer quelques explications à titre exploratoire. Des études menées sur l'évaluation des habiletés sociales de jeunes de niveau scolaire ont révélé que, lorsqu'on les compare à l'aide de corrélations, les perceptions des adultes (parents, intervenants scolaires) diffèrent de celles des élèves (Achenbach, McConaughy \& Howell, 1987; DiPerna \& Volpe, 2005; Junttila et al., 2006) ${ }^{5}$. La formule autorévélée pour évaluer le comportement ou les habiletés sociales d'un élève procure une information unique reposant sur sa propre perception; cependant, la nature subjective de cette technique exige que la procédure d'évaluation comprenne d'autres sources d'information (Warnes et al., 2005). Selon Cartledge et Milburn (1995), l'autoévaluation faite par l'élève peut servir à fournir une information précieuse sur les différences de perceptions par rapport aux autres sources d'information, à amorcer une prise de conscience avec l'élève sur ces divergences et à orienter les objectifs de changement de comportement par l'intervention. Aussi, selon Junttila et collaborateurs (2006), la thèse du lien affectif des parents pour leur enfant venant considérablement influencer leur évaluation pourrait expliquer les écarts entre les perceptions des parents et celles des intervenants scolaires. À ce chapitre, il est à remarquer que les parents et les intervenants scolaires observent les comportements des enfants dans des contextes très différents. Puisque le comportement des enfants est susceptible de varier considérablement en fonction des contextes, il n'est pas surprenant de constater des écarts quant à leurs perceptions respectives. Notons que Cartledge et Milburn (1995) ont aussi trouvé que le recours à une procédure multisources d'information aurait pour effet d'augmenter la précision des résultats de l'évaluation du comportement.

Avant de planifier et de mettre en œuvre une intervention visant à modifier des comportements ou des habiletés sociales chez des élèves, il est nécessaire de procéder à une évaluation rigoureuse du répertoire comportementale (forces et besoins) afin de déterminer si un nouveau comportement doit être enseigné ou simplement rappelé (Cartledge \& Milburn, 1995). Ces informations peuvent influencer grandement le choix de l'approche et de la programmation de l'intervention et donc avoir des retombées positives sur l'efficacité de la démarche et ses divers acteurs (élèves, parents, intervenants scolaires et sociaux) (Flanagan et al., 1996; Warnes et al., 2005). Les succès du procédé ayant recours aux multiples sources d'information, à plusieurs indicateurs du niveau d'habiletés sociales et à plusieurs temps de mesure pour évaluer avec précision les habiletés sociales d'adolescents ayant des troubles du comportement confirment que les chercheurs et les praticiens doivent investir des ressources en ce sens. Comme l'intervention initiale auprès de ces jeunes nécessite un investissement considérable, il est d'autant plus important de disposer d'informations fidèles et valides pour orienter les choix s'y rattachant.

\footnotetext{
${ }^{5}$ Pour ce qui est de la présente étude, des corrélations de l'ordre de 0,02 à 0,60 ont été obtenues lorsqu'on a comparé les perceptions des parents et celles des adolescents pour chacun des indicateurs du niveau d'habiletés sociales, à chacun des temps de mesure. Pour la comparaison des perceptions des intervenants scolaires et celles des adolescents, les corrélations ont varié de $-0,32$ à 0,51 .
} 


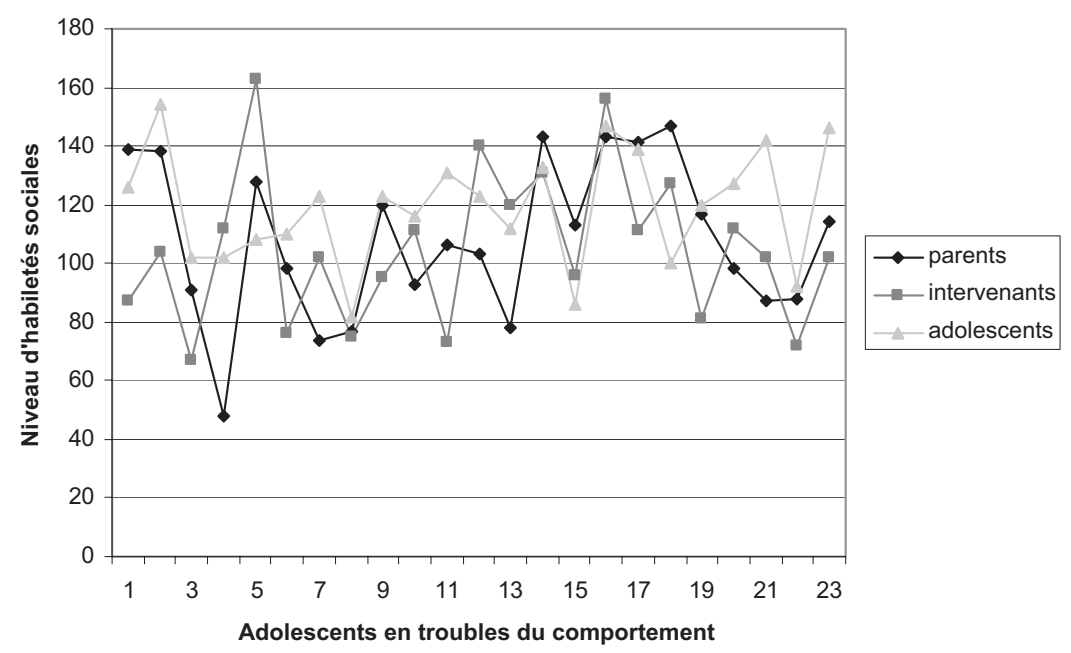

Figure 2. Illustration de l'interaction Adolescents ayant des troubles du comportement $\times$ Sources d'information.

\section{Conclusion}

L'objectif de cette recherche était d'évaluer la fiabilité d'un dispositif d'évaluation des habiletés sociales chez des adolescents ayant des troubles du comportement. Les résultats ont permis de confirmer la fiabilité du dispositif d'évaluation visant à assurer la différenciation des adolescents ayant des troubles du comportement selon leur niveau d'habiletés sociales, et ce, sans égard au temps de mesure, à la source d'information ou à l'indicateur du niveau d'habiletés sociales privilégié.

Bien que des recherches futures soient nécessaires, le dispositif d'évaluation préconisé dans cette étude permet de pallier les trois limites énoncées par des chercheurs (Cartledge \& Milburn, 1995; Flanagan et al., 1996; Gresham, 1998). Tout d'abord, le dispositif repose sur l'utilisation d'un outil de mesure largement utilisé et validé auprès d'un échantillon québécois (Questionnaire d'évaluation des habiletés sociales de Fortin et al., 2001; traduction du Social Skills Rating System - SSRS -, de Gresham \& Elliott,
1990). Ensuite, ce questionnaire a l'avantage de recueillir la perception de différentes sources d'information (adolescent, parent, intervenant scolaire). Enfin, une évaluation rigoureuse dans le temps est effectuée avec un prétest et deux postests.

La présente étude comportait certaines limites qu'il importe de mentionner, notamment en ce qui a trait à la théorie de la généralisabilité et au logiciel utilisé pour évaluer la fiabilité du dispositif d'évaluation considéré. D'abord, le fait que le logiciel EduG (version 1.3) ne permette pas l'utilisation de devis non balancé a eu pour conséquence de retirer des analyses de généralisabilité les éléments suivants : 1) les sous-échelles « empathie » et « responsabilisation » du Questionnaire d'évaluation des habiletés sociales, car elles ne s'adressent qu'aux adolescents et aux parents respectivement; 2) les adolescents pour lesquels chacun des indicateurs du niveau d'habiletés sociales n'a pas été apprécié par chacune des sources d'information à chacun des temps de mesure. Ensuite, il appert

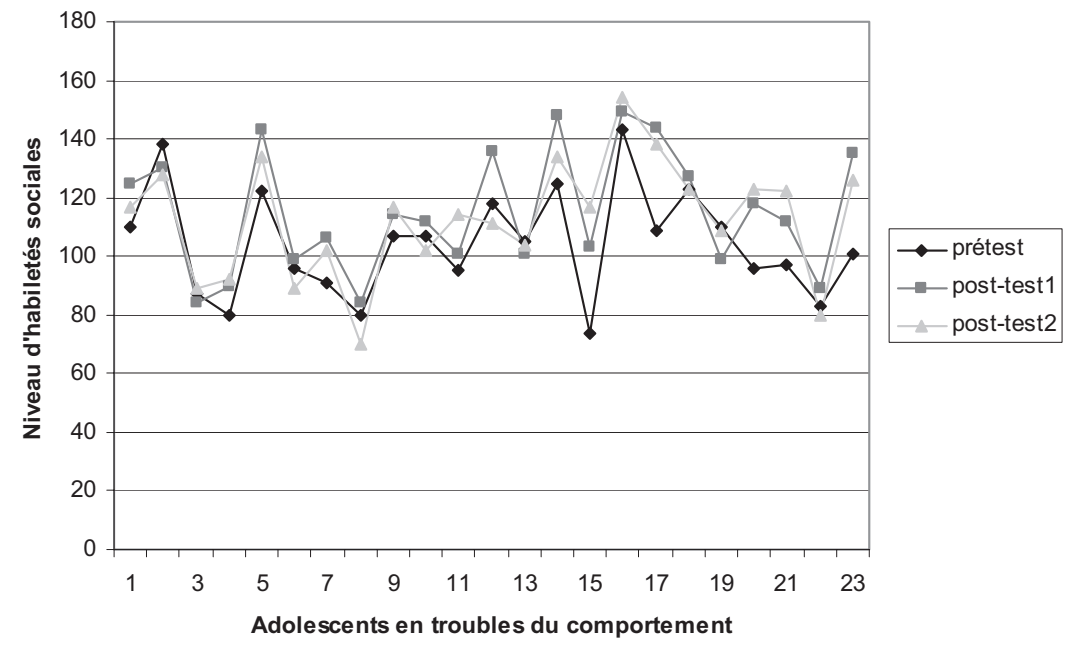

Figure 3. Illustration de l'interaction Adolescents ayant des troubles du comportement $\times$ Temps de mesure. 


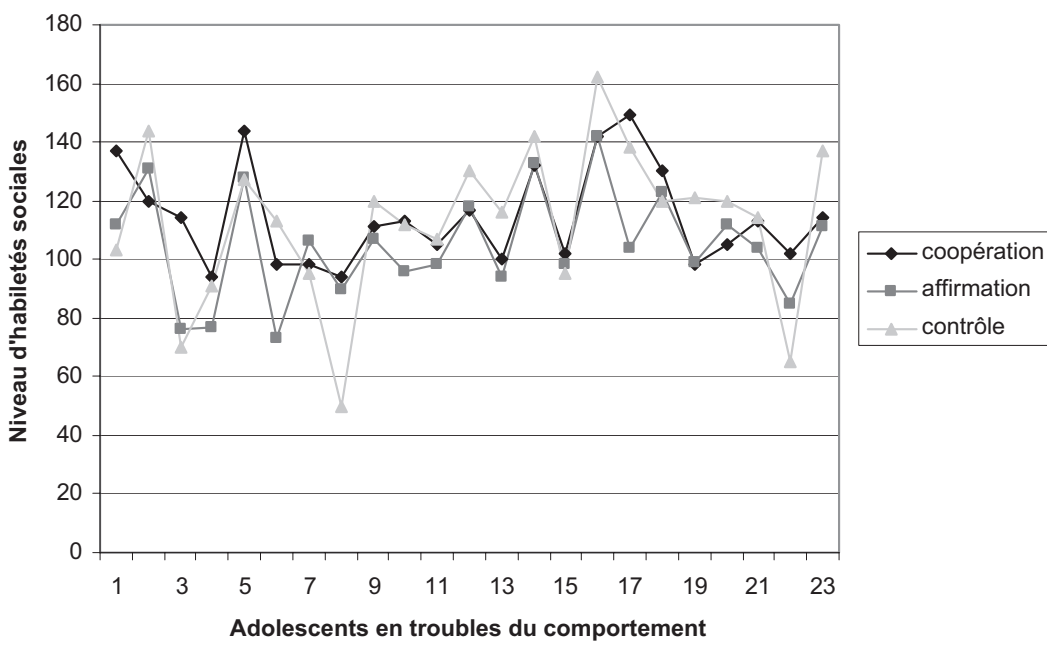

Figure 4. Illustration de l'interaction Adolescents ayant des troubles du comportement $\times$ Indicateurs du niveau d'habiletés sociales.

que le nombre d'adolescents ayant des troubles du comportement échantillonnés (23) ait été quelque peu limité pour les analyses de généralisabilité effectuées. Ceci dit, malgré le bienfondé de cette limite, le fait que les erreurs-type des composantes de variance soient petites (Bertrand \& Blais, 2004) met en lumière l'impact minime qu'a eu l'échantillon restreint sur la précision de la mesure fournie par le dispositif d'évaluation. Toute technique d'évaluation comportementale ayant ses limites (Cartledge \& Milburn, 1995; Warnes et al., 2005), il serait intéressant de jumeler un outil multisources d'information comme le SSRS (à savoir, une échelle normative) et une grille d'observation prenant aussi en compte l'aspect contextuel du comportement (à savoir, une échelle fonctionnelle). Enfin, il y a lieu de mentionner l'absence d'information sur la nature et la gravité des problèmes de comportements chez les adolescents.

\footnotetext{
Abstract

Behaviour difficulties (BD) in school-age children and adolescent constitute an increasingly important concern for all participants in the Quebec and foreign education systems. The risk factors that can contribute to the development of BD are numerous (Kauffman, 2005). Amongst these appears a whole set of skills related to social competence (Bowen, Desbiens, Martin, \& Hamel, 2001). The present study aims to test, by means of Generalisability theory, the reliability of the evaluation process developed to measure the level of teenage social skills according to various indicators (cooperation, self-control, assertion), various sources of information (adolescent, parent, and teacher), and various times (pretest, posttest 1, posttest 2). The coefficient of relative generalisability was found to be higher than the limit considered satisfactory, indicating that the evaluation process is reliable enough to allow differentiation of social skills for adolescent with BD. To develop intervention guidelines for BD students, a review of the sources of variation that may influence the accuracy of the measurement provided by the evaluation process is conducted through an analysis of facets
}

(Bertrand \& Blais, 2004) and a study of the most important variances components (Bain \& Pini, 1996).

Keywords: generalisability, social skills, adolescent

\section{Références}

Achenbach, T. M., McConaughy, S. H., \& Howell, C. T. (1987). Child/ adolescent behavioral and emotional problems: Implications of crossinformant correlations for situational specificity. Psychological Bulletin, 101, 213-232.

American Psychiatric Association. (1994). Diagnostic and statistical manual of mental disorders (4th ed.). Washington, DC : American Psychiatric Association.

Bain, D., \& Pini, G. (1996). La généralisabilité : mode d'emploi. Genève, Suisse : Centre de recherches psychopédagogiques.

Bandura, A. (1986). Social foundations of thought and action: A social cognitive theory. Englewood Cliffs, NJ : Prentice Hall.

Bertrand, R. (1988). Pourquoi de nouvelles théories de la mesure? Mesure et évaluation en éducation, 11, 5-25.

Bertrand, R., \& Blais, J. (2004). Modèles de mesure : l'apport de la théorie des réponses aux items. Ste-Foy, Québec : Presses de l'Université du Québec.

Bowen, F., Desbiens, N., Gendron, M., \& Bélanger, J. (2006). L'acquisition et le développement des habiletés sociales. Dans L. Massé, N. Desbiens, \& C. Lanaris (éds), Les troubles de comportements à l'école : diagnostic et intervention (pp. 213-227). Montréal : Gaëtan Morin Éditeur Ltée.

Bowen, F., Desbiens, N., Martin, C., \& Hamel, M. (2001). La compétence sociale. Dans Gouvernement du Québec (éd.), 61217 Nous serons bien mieux! Les déterminants de la santé et du bien-être des enfants d'âge scolaire (pp. 55-99). Québec : Les Publications du Québec.

Cardinet, J., \& Tourneur, Y. (1985). Assurer la mesure. Berne, Suisse : Peter Lang.

Cartledge, G., \& Milburn, J. F. (1995). Teaching social skills to children and youth: Innovative approaches (3rd ed.). Toronto : Allyn \& Bacon.

Cloutier, R. (1996). Psychologie de l'adolescence ( $2^{\mathrm{e}}$ éd.). Montréal : Gaëtan Morin Éditeur Ltée.

Diperna, J. C., \& Volpe, R. J. (2005). Self-report on the social rating 
system analysis of reliability and validity for an elementary sample. Psychology in the Schools, 42, 345-354.

Doucette, B. W., \& Fowler, S. M. (1997). PACTE : Niveaux 7-12 : un programme de développement d'habiletés socio-affectives. Montréal : Chenelière/McGraw-Hill.

EduG (Version 1.3), logiciel informatique. (2002). Lévis, Québec : EDUCAN. Flanagan, D. P., Alphonso, V. C., Primavera, L. H., Povall, L., \& Higgins, D. (1996). Convergent validity of the BASC and SSRS: Implications for social skills assessment. Psychology in the Schools, 33, 13-23.

Fortin, L., Royer, E., Marcotte, D., \& Potvin, P. (2000). Les facteurs discriminants sur les plans personnel, familial et scolaire entre les troubles du comportement intériorisés, extériorisés et concomitants chez des élèves de première secondaire. Revue des sciences de l'éducation, 26, 197-218.

Fortin, L., Royer, É., Marcotte, D., Potvin, P., \& Joly, J. (2001). Épreuves de validité d'une mesure d'habiletés sociales auprès d'adolescents québécois à l'école secondaire. Psychologie et Psychométrie, 22, 23-44.

Gagnon, C., Boisjoli, R., Gendreau, P. L., \& Vitaro, F. (2006). Le trouble oppositionnel avec provocation et trouble des conduites. Dans L. Massé, N. Desbiens, \& C. Lanaris (éds), Les troubles de comportements à l'école : diagnostic et intervention (pp. 17-27). Montréal : Gaëtan Morin Éditeur Ltée.

Gendron, M., Royer, É., Bertrand, R., \& Potvin, P. (2005). Les troubles du comportement, la compétence sociale et la pratique d'activités physiques chez les adolescents. Revue des sciences de l'éducation, 31, 211-233.

Gendron, M., Royer, É., Bertrand, R., Potvin, P., \& Frenette, É. (2006). Relation entre le sentiment d'auto-efficacité, les habitudes de vie et les habiletés sociales chez les adolescents en difficulté de comportement. Revue de psychoéducation, 35, 229-250.

Gendron, M., Royer, E., \& Morand, C. (2005). Programme PEC - Pratiquons ensemble nos compétences : Guide II - Volet scolaire. Québec : Centre de santé de Portneuf.

Gendron, M., Royer, É., Potvin, P., \& Bertrand, R. (2003). Troubles du comportement, compétence sociale et pratique d'activités physiques chez les adolescents : enjeux et perspectives d'intervention. Revue de psycho-éducation, 32, 349-372.

Goldstein, A. P., \& McGinnis, E. (1997). Skillstreaming the Adolescent: New strategies and perspectives for teaching prosocial skills. Champaign, IL : Research Press.

Goupil, G. (2007). Les élèves en difficulté d'adaptation et d'apprentissage ( $3^{\mathrm{e}}$ éd.). Boucherville, Québec : Gaétan Morin Éditeur.

Gresham, F. M. (1998). Social skills training: Should we raze, remodel, or rebuild? Behavioral Disorders, 24, 19-25.
Gresham, F. M., \& Elliott, S. N. (1990). Social Skills Rating System manual. Circle Pines, MN : American Guidance Service.

Junttila, N., Voeten, M., Kaukiainen, A., \& Vaurus, M. (2006). Multisource assessment of children's social competence. Educational and Psychological Measurement, 66, 874-895.

Kauffman, J. M. (2005). Characteristics of emotional and behavioral disorders of children and youth (8th ed.). Upper Saddle River, NJ : Pearson Prentice Hall.

Ministère de l'Éducation du Québec (1999). Une école adaptée à tous ses élèves : Prendre le virage du succès. Politique de l'adaptation scolaire. Québec : Ministère de l'éducation.

Ministère de l'Éducation du Québec. (2000). Élèves handicapés ou élèves en difficulté d'adaptation ou d'apprentissage (EHDAA) : Définitions. Québec : Ministère de l'éducation.

Potvin, P. (dir.) (1994). Prends le volant : programme pour développer les habiletés sociales et l'autocontrôle des adolescents ayant des troubles du comportement. Collaborateurs : Ministère de l'Éducation; Direction régionale de la Mauricie-Bois-Francs. Trois-Rivières, Québec : Commission scolaire de Trois-Rivières.

Potvin, P. (dir.) (1995). PARC : programme d'autocontrôle, de résolution de problèmes et de compétence sociale pour les élèves du primaire ayant des troubles du comportement. Collaborateurs : Ministère de l'Éducation; Direction régionale de la Mauricie-Bois-Francs. Trois-Rivières, Québec : Commission scolaire de Trois-Rivières.

Saint-Laurent, L. (2008). Enseigner aux élèves à risque et en difficulté au primaire ( $2^{\mathrm{e}}$ éd.). Boucherville, Québec : Gaëtan Morin ÉditeurChenelière Éducation.

Tremblay, R. (1998). Les élèves qui présentent une difficulté de comportement. Revue québécoise de psychologie, 19, 103-124.

Vitaro, F., \& Gagnon, C. (2000). Prévention des problèmes d'adaptation chez les enfants et les adolescents - Tome II : les problèmes externalisés. Sainte-Foy, Québec : Presses de l'Université du Québec.

Walker, H. M., Colvin, G., \& Ramsey, E. (1995). Antisocial behaviour in school: Strategies and best practices. Toronto : Brooks/Cole.

Warnes, E. D., Sheridan, S. M., Geske, J., \& Warnes, W. A. (2005). A contextual approach to the assessment of social skills: Identifying meaningful behaviours for social competence. Psychology in the Schools, 42, 173-187.

Reçu le 1 janvier 2007

Révisé le 25 mars 2009

Accepté le 26 mars 2009 